\title{
Uniqueness of Padé Approximants From Series of Orthogonal Polynomials
}

\section{By Avram Sidi}

\begin{abstract}
It is proved that whenever a nonlinear Padé approximant, derived from a series of orthogonal polynomials, exists, it is unique.
\end{abstract}

Let $\phi_{r}(x), r=0,1,2, \ldots$, be a set of polynomials which are orthogonal on an interval $[a, b]$, finite, semi-infinite, or infinite, with weight function $w(x)$, whose integral over any subinterval of $[a, b]$ is positive; i.e.,

$$
\int_{a}^{b} w(x) \phi_{r}(x) \phi_{s}(x) d x=0 \text { if } r \neq s .
$$

Then it is known that $\phi_{r}(x)$ is a polynomial of degree exactly $r$.

Suppose now $f(x)$ is a function which has a formal expansion of the form

$$
f(x)=\sum_{r=0}^{\infty} a_{r} \phi_{r}(x)
$$

on $[a, b]$. The $(m, n)$ Padé approximant to $f(x)$ is defined to be the rational function

$$
S_{m, n}(x)=\frac{P(x)}{Q(x)}=\frac{\sum_{r=0}^{m} p_{r} \phi_{r}(x)}{\sum_{s=0}^{n} q_{s} \phi_{s}(x)}
$$

having an expansion in $\phi_{r}(x), r=0,1,2, \ldots$, which agrees with that of $f(x)$ given in (2) up to and including the term $a_{m+n} \phi_{m+n}(x)$. It is assumed that the polynomials $P(x)$ and $Q(x)$ have no common factor, apart from a constant, and that $Q(x)$ does not vanish on $[a, b]$. It is worth mentioning that the approximations defined above are the ones called "nonlinear Padé approximants" in [2] .

THEOREM 1. If $g(x)$ is any continuous function on $[a, b]$ such that $\int_{a}^{b} w(x) g(x) \phi_{r}(x) d x=0, r=0,1, \ldots, k-1$, then $g(x)$ either changes sign at least $k$ times in the interval $(a, b)$ or is identically zero.

The proof of this theorem can be found in $[1$, p. 110].

As a consequence of Theorem 1 , it follows that if $Q(x)$ is nonzero on $[a, b]$, then $q_{0} \neq 0$; hence one can normalize $Q(x)$ by taking $q_{0}=1$.

Theorem 2. If the $(m, n)$ th nonlinear Pade approximant $P(x) / Q(x)$ to $f$ exists, in the sense of (3), and, after dividing out common factors, if $Q$ is of one sign on $[a, b]$, then it is unique.

Proof. By the definition of $S_{m, n}(x)=P(x) / Q(x)$ one has

$$
f(x)-S_{m, n}(x)=\sum_{r=m+n+1}^{\infty} A_{r} \phi_{r}(x) .
$$


If $\bar{S}_{m, n}(x)=\bar{P}(x) / \bar{Q}(x)$ is another $(m, n)$ Padé approximant to $(1)$, then

$$
f(x)-\bar{S}_{m, n}(x)=\sum_{r=m+n+1}^{\infty} \bar{A}_{r} \phi_{r}(x) .
$$

Subtracting (4) from (5) one obtains

$$
S_{m, n}(x)-\bar{S}_{m, n}(x)=\sum_{r=m+n+1}^{\infty}\left(\bar{A}_{r}-A_{r}\right) \phi_{r}(x) .
$$

Now since $S_{m, n}(x)$ and $\bar{S}_{m, n}(x)$ are continuous on $[a, b]$ so is $D(x) \equiv S_{m, n}(x)$ $\bar{S}_{m, n}(x)$. Then from (6) it follows that $D(x)$ satisfies $\int_{a}^{b} w(x) D(x) \phi_{r}(x) d x=0, r=$ $0,1, \ldots, m+n$. Hence by Theorem $1, D(x)$ either changes sign at least $m+n+1$ times on $(a, b)$, or is identically zero there. But

$$
D(x)=\frac{P(x)}{Q(x)}-\frac{\bar{P}(x)}{\bar{Q}(x)}=\frac{P(x) \bar{Q}(x)-\bar{P}(x) Q(x)}{Q(x) \bar{Q}(x)},
$$

i.e., the numerator of $D(x)$ is a polynomial of degree at most $m+n$, therefore, can have at most $m+n$ zeros on $(a, b)$. Since $Q(x)$ and $\bar{Q}(x)$ are nonzero on $[a, b], D(x)$ changes sign at most $m+n$ times on $(a, b)$. Therefore, $D(x) \equiv 0$; hence $S_{m, n}(x) \equiv$ $\bar{S}_{m, n}(x)$. Q.E.D.

So far Padé approximants from Legendre series [2] and Chebyshev series have been considered [3], [4]. As is explained in [2], the determination of the $q_{s}, s=1$, $2, \ldots, n$, in general, involves the solution of $n$ nonlinear equations, the determination of the $p_{r}$ being trivial then. However, these $n$ equations may have several solutions. But, as is mentioned in [2], only one solution with $Q(x) \neq 0$ on $[a, b]$ has been found for the examples in [2]. By Theorem 2 there is no other solution, and it is at this point that the result of Theorem 2 becomes important.

The author wishes to thank Professor I. M. Longman for encouragement and help.

Department of Geophysics and Planetary Sciences

Tel-Aviv University

Ramat-Aviv, Israel

1. E. W. CHENEY, Introduction to Approximation Theory, McGraw-Hill, New York, 1966. MR 36 \#5568.

2. J. FLEISCHER, "Nonlinear Padé approximants for Legendre series," J. Mathematical Phys., v. 14, 1973, pp. 246-248. MR 48 \#592.

3. C. W. CLENSHAW \& K. LORD, "Rational approximations from Chebyshev series," Studies in Numerical Analysis, Academic Press, London, 1974, pp. 95-113. MR 50 \#8914.

4. A. SIDI, "Computation of the Chebyshev-Padé table," J. Comput. Appl. Math., v. 1, 1975, pp. 69-71. MR $52 \# 4580$. 